\title{
Experimental investigation on flow structures of steadily translating low-aspect-ratio wings in low Reynolds number flow
}

\author{
Yichen Zhu ${ }^{1}$, Jinjun Wang ${ }^{1 *}$ \\ ${ }^{1}$ Beijing University of Aeronautics and Astronautics, Key Laboratory of Fluid Mechanics (Ministry \\ of Education), Beijing, China \\ *jjwang@buaa.edu.cn
}

\begin{abstract}
In recent decades, Micro Air Vehicles (MAVs) have been a hot topic for their promising future. But the promotions of MAVs are hindered by their short endurances. To solve this problem, inspirations are brought from migratory butterflies who utilize the 'flapping-gliding' skill during long-distance migration to improve the flight efficiency. The butterfly's gliding flights, which can be simplified by considering the steadily translating fixed wings, have drawn high attentions. Previous studies mainly focus on the aerodynamics of the low-aspect-ratio fixed wings at $R e \approx 10^{5}$ via force measurements. However, few experimental studies have measured the 3D flow fields. Consequently, the underlying high lift-to-drag ratio mechanisms in the steadily translating butterfly-shaped wings are still not clear. To shed new light on this problem, the 3D flow structures around butterfly-shaped wings were captured and investigated in detail.

In the present work, experiments were conducted in a low-speed recirculation water channel at Beijing University of Aeronautics and Astronautics. The channel has a test section of $60 \mathrm{~cm}$ (height) $\times$ $60 \mathrm{~cm}$ (width) $\times 300 \mathrm{~cm}$ (length). Three kinds of flat-plate models were tested in our experiments. Fig. 1 shows the schematics of the wing planforms. The outline of the Danaus plexippus model (DP, Fig. 1(a)) was derived from the typical museum specimen of the corresponding butterfly species. This planform has been used in our previous study(Zhu et al. (2020)). To better understand the effects of wing planform, two simplified models were also investigated. The models with forward-sweep and straight leading edges were called the forward-sweep trapezoid model (FST, Fig. 1(b)) and the nonesweep trapezoid model (NST, Fig. 1(c)), respectively. The chord, span and thickness of the models were $l=80 \mathrm{~mm}, c=60 \mathrm{~mm}$ and $t=1 \mathrm{~mm}$, respectively. All edges were left square. The models were suspended upside down into the test section from a strut system which can control the Angle of Attacks (AoAs, $\alpha$ ) precisely. The AoAs varied from $4^{\circ}$ to $10^{\circ}$ with a step of $2^{\circ}$ in our study. The strut system was installed on a robotic translator, which can translate the models along the freestream direction. The free-stream velocity was $U_{\infty}=100 \mathrm{mms}^{-1}$ resulting in a chord-based Reynolds number $R e_{c}=5.4 \times 10^{3}$. The free-stream turbulent intensity was less than $1 \%$. The Stereo-PIV measurements were employed to obtain the three-component velocity fields with a fixed laser sheet placed perpendicularly to the free-stream direction. The models were translated via robotic translator in increments of $\Delta x=0.05 c$. Thus, the approximations of the time-averaged volumetric flow fields were reconstructed by the multiple-plane data. The coordinate system used here originates at the trailing edge of the models at the midspan location, where $\mathrm{X}, \mathrm{Y}$ and $\mathrm{Z}$ axes refer to the streamwise, spanwise and normal directions, respectively. The measurement volume was in the coordinate range of $[-c, 0.5 c] \times[-0.75 c, 0.75 c] \times[-0.25 c, 0.5 c]$. In order to investigate the evolutions of flow structures, the time-resolved 2D2C PIV measurements were also performed with a laser sheet placed perpendicularly to the spanwise direction. For each case, two independent sets of 13431 instantaneous velocity fields were acquired at a sampling rate of $288 \mathrm{~Hz}$.

The analysis begins by examining the 3D time-averaged flow structures of the three models. The representative flow fields of these models at $\alpha=8^{\circ}$ are shown in Fig. 1 . The vorticity magnitudes $\left\|\omega c / U_{\infty}\right\|_{2}$ are used to visualize the vortex sheets where vortex cores are identified by an isosurface of Q-critierion $(Q=43)$. In the leeward side of the DP and FST models, two elongate vortical structures are identified by $Q$, which represent the leading-edge vortices (LEVs) and might be casused
\end{abstract}


by the presence of curved leading edges. The LEVs remain attached to these two models, which are probably the sources of additional non-linear lift. However, the LEVs cannot be identified from the time-averaged flow fields for the cases of NST model. In fact, the individual LEV convects downstream once detaching from the leading-edge shear layer. The temporal-spatial evolutions of the LEVs are reconstructed based on the long short term memory (LSTM) method. In addition to the LEVs, the wingtip vortices (WTVs) are identified for each case in Fig. 1, as a pair of counter-rotating vortices trailing from the wing tips. The instantaneous locations of WTV centers are determined by $\Gamma_{1}$ criterion, which is recently used by Dghim et al. (2020). The detected $\Gamma_{1}$ peaks are fitted by Gaussian model to locate WTV centers more precisely. The mean locations of WTV centers are then carculated and fitted by straight lines in the streamwise range of $x / c=[-0.65,0.5]$. Fig. 2 shows the detected results at $\alpha=10^{\circ}$. Furthermore, the WTV parameters such as trajectory,core radius and core circulation are obtained. It is found that the WTV trajectories move inward and downward with increasing AOAs regardless of the wing planforms. To compare the effects of the wing planforms quantitatively, the induced drag coefficients $C_{D i}$ are calculated based on the Maskell induced-drag model. The variations of $C_{D i}$ with $\alpha$ are shown in Fig. 3. Interestingly, the DP model has the smallest $C_{D i}$ of the three models in the range of $\alpha=\left[4^{\circ}, 10^{\circ}\right]$. Thus, it is likely that the butterfly-shaped wings have relatively small induced drags, which contribute to improve the high lift-to-drag ratio.

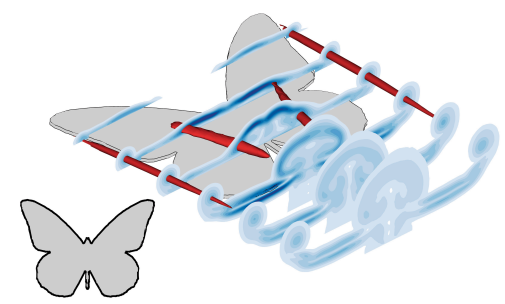

(a) DP model

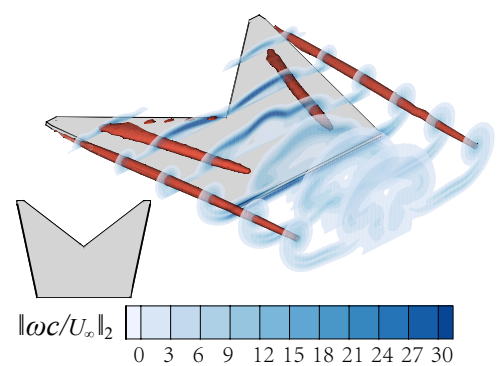

(b) FST model

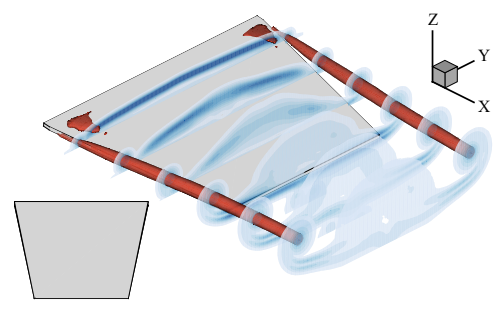

(c) NST model

Figure 1: Perspective view of the time-averaged flow fields at $\alpha=8^{\circ}$. Shown in red is the isosurface of $Q=43$.The planforms are also sketched at the bottom left.

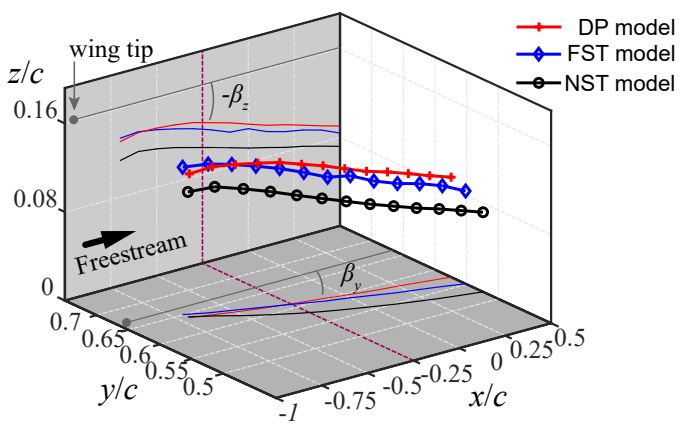

Figure 2: Mean locations of WTV centers with $x / c$ in the rang of $[-0.65,0.5]$ at $\alpha=10^{\circ}$

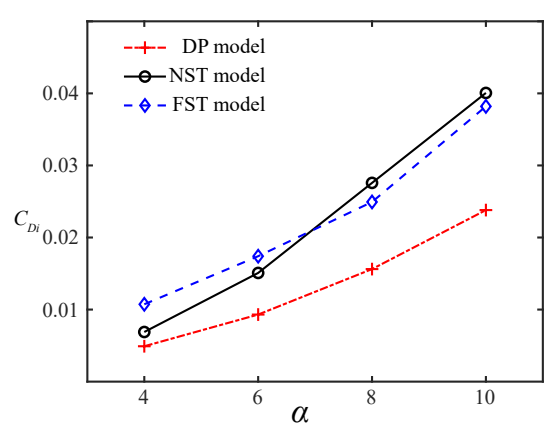

Figure 3: Variations of $C_{D i}$ with $\alpha$.

\section{References}

Dghim M, Ferchichi M, and Fellouah H (2020) On the effect of active flow control on the meandering of a wing-tip vortex. Journal of Fluid Mechanics 896:A30

Zhu Y, Qu Y, Wang J, and Ma B (2020) Near-wall topological patterns and flow structures over a simplified Danaus plexippus model. Chinese Journal of Aeronautics 33:2527-2534 